\title{
Subdominant pairing channels in unconventional superconductors: Ginzburg-Landau theory
}

\author{
Qiang-Hua Wang \\ Department of Physics and National Laboratory of Solid State Microstructures, Institute for Solid State Physics, \\ Nanjing University, Nanjing 210093, China \\ and Department of Physics, University of Hong Kong, Pokfulam Road, Hong Kong, China \\ Z. D. Wang* and Q. Li \\ Department of Physics, University of Hong Kong, Pokfulam Road, Hong Kong, China
}

(Received 19 March 1999)

\begin{abstract}
A Ginzburg-Landau theory is developed for unconventional superconductors with the three relevant singlet pairing channels (i.e., $s, d_{x^{2}-y^{2}}$, and $d_{x y}$ channels). Various consequences of the subdominant channels (i.e., $s$ and $d_{x y}$ channels) are examined in detail. (1) In the case of a $d_{x^{2}-y^{2}}+i s$-wave superconductor, we reproduce an earlier result that there is a second-order zero-field transition from the pure $d_{x^{2}-y^{2}}$ phase to the timereversal-symmetry- ( $T_{-}$) breaking $d_{x^{2}-y^{2}}+i s$-phase at the temperature $T_{D S}$. The structure of a single vortex above and below $T_{D S}$ is fourfold and twofold symmetric, respectively. (2) In the case of a $d_{x^{2}-y^{2}}+i d_{x y}$-wave superconductor, there is also a second-order zero-field phase transition from the pure $d_{x^{2}-y^{2}}$ phase to the T-breaking $d_{x^{2}-y^{2}}+i d_{x y}$-wave phase at the temperature $T_{D D^{\prime}}$. In contrast to the case in a $d_{x^{2}-y^{2}}+i s$-wave superconductor, the subdominant phase cannot be induced by vortices above $T_{D D^{\prime}}$. Below the $T$-breaking transition, the subdominant phase in the mixed state is nontrivial: it survives at low fields, but may disappear above a field (increasing with decreasing temperature) presumably via a first-order transition. (3) By including the strong-coupling effects, a $T$-breaking-coupling term between the $d_{x^{2}-y^{2}}$ and $d_{x y}$ waves is found to have significant effects on the low-temperature behavior of $d_{x^{2}-y^{2}}+i d_{x y}$ superconductors. In a magnetic field, a $d_{x^{2}-y^{2}}+i d_{x y}$ state is always established, but the field dependence of $d_{x y}$ amplitude above $T_{D D^{\prime}}$ is different from that below $T_{D D^{\prime}}$. Above but not very close to $T_{D D^{\prime}}$, the induced minimum gap $\Delta_{0} \propto B /\left(T-T_{D D^{\prime}}\right)$.
\end{abstract} [S0163-1829(99)01446-0]

\section{INTRODUCTION}

The phase-sensitive experiment of the unusual Josephson effect in high- $T_{c}$ superconductors evidenced the

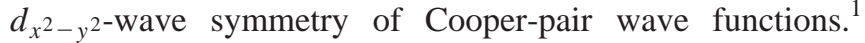
Thus, at least the problem as what is the dominant channel for electrons to pair in high- $T_{c}$ superconductors has been resolved. However, a new puzzle arises as what is the subdominant pairing channel, which seems to have manifested in a number of experiments, e.g., the observation of surfaceinduced broken time-reversal symmetry ( $T$ hereafter) in YBCO tunnel junctions, ${ }^{2}$ the observation of fractional vortices trapped in a boundary junction, ${ }^{3}$ and the abnormal field dependence of the low-temperature thermal conductivity $\kappa_{e}$ in $\mathrm{BSCCO},{ }^{4,5}$ to name only a few. At surfaces, the subdominant channel can show up because of a suppression of the effective critical temperature in the dominant $d_{x^{2}-y^{2}}$ channel. Rather differently, the anomalous $\kappa_{e}$ reported in Ref. 4 might require a mechanism to produce bulk $T$-breaking states. ${ }^{6,7}$ This scenario might work above $5 \mathrm{~K}$ in the samples reported in Refs. 4,5 but failed to explain the data at subKelvin temperatures in Ref. 5. Instead, field-induced quasi-

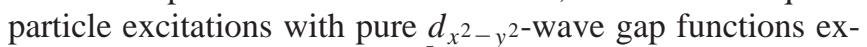
plain qualitatively these data. ${ }^{5}$

As a modeling study, we consider the relevant singlet subdominant pairing channels in an unconventional $d$ superconductor to be the $s$ and $d_{x y}$ channels, which may be relevant to high temperature superconductors. For attractive pairing interactions in the $s$ and $d_{x^{2}-y^{2}}$ channels, or in the $d_{x^{2}-y^{2}}$ and $d_{x y}$ channels, the uniform superconducting state in the absence of magnetic field has been shown to be either a pure state in the dominant channel, or a state with a broken time-reversal symmetry involving the relevant channels. ${ }^{7,8} \mathrm{In}$ order to study the vortex states, we develop a GinzburgLandau (GL) theory that includes the above-mentioned subdominant channels. We discuss the consequences of these channels on the properties of the superconductors, such as the field dependence of the thermal conductivity. The structure of this paper is as follows. The microscopic derivation of the GL theory (in the weak-coupling limit) is presented in Sec. II. The properties of $d_{x^{2}-y^{2}}+i$ s superconductors are described in Sec. III. The properties of $d_{x^{2}-y^{2}}+i d_{x y}$ superconductors are addressed in Sec. IV. A phenomenological strong-coupling correction to the weak-coupling theory is developed and discussed in Sec. V with respect to the $d_{x^{2}-y^{2}}$ $+i d_{x y}$ superconductors. Section VI contains a summary and some concluding remarks.

\section{DERIVATION OF THE GINZBURG-LANDAU THEORY IN THE WEAK-COUPLING LIMIT}

The simplest way to derive microscopically a GinzburgLandau theory for superconductors is to consider the Bardeen-Cooper-Shrieffer (BCS) gap equation. At zero center-of-mass momentum for the Cooper pairs, the gap function is 


$$
\Delta_{k}=\sum_{k^{\prime}} V_{k, k^{\prime}}\left\langle C_{-k^{\prime}, \downarrow} C_{k^{\prime}, \uparrow}\right\rangle=\sum_{k^{\prime}} \frac{V_{k, k^{\prime}} \Delta_{k^{\prime}}}{2 E_{k^{\prime}}} \tanh \frac{\beta E_{k^{\prime}}}{2},
$$

which follows routinely from the effective BCS Hamiltonian. Here $E_{k}=\sqrt{\epsilon_{k}^{2}+\left|\Delta_{k}\right|^{2}}$ is the quasiparticle excitation spectrum and $\beta=1 / T$ is the inverse temperature. (We are using the units $k_{B}=\hbar=c=1$, and are measuring energies from the Fermi surface.) In the weak-coupling limit the summation over momentum in the gap equation is understood to be restricted near the Fermi surface. Before going into further details, we need to specify the pairing interaction $V_{k, k^{\prime}}$. Due to the tetragonal symmetry of the copper planes, it is rather reasonable to assume the following form for $V_{k, k^{\prime}}$,

$$
\begin{aligned}
V_{k, k^{\prime}} & =V_{s}+V_{x^{2}-y^{2}}\left(\hat{k}_{x}^{2}-\hat{k}_{y}^{2}\right)\left(\hat{k}_{x}^{\prime 2}-\hat{k}_{y}^{\prime 2}\right)+4 V_{x y} \hat{k}_{x} \hat{k}_{y} \hat{k}_{x}^{\prime} \hat{k}_{y}^{\prime} \\
& =V_{s}+V_{x^{2}-y^{2}} \cos 2 \theta_{k} \cos 2 \theta_{k^{\prime}}+V_{x y} \sin 2 \theta_{k} \sin 2 \theta_{k^{\prime}} .
\end{aligned}
$$

Henceforth, the subscripts $s, x^{2}-y^{2}$, and $x y$ are channel indices referring to the $s, d_{x^{2}-y^{2}}$, and $d_{x y}$ channels of the pairing interaction, respectively. $\theta_{k}$ is the angle between the wave vector $k$ and the $a$ axis in the copper-oxide plane. By symmetry, the gap function can be expressed as

$$
\Delta_{k}=S+D \cos 2 \theta_{k}+D^{\prime} \sin 2 \theta_{k},
$$

where $S, D$, and $D^{\prime}$ are complex amplitudes of the gap function in the $s, d_{x^{2}-y^{2}}$, and $d_{x y}$ channels, respectively, and are also called as the order parameters. Inserting Eqs. (2) and (3) into Eq. (1), expanding the gap equation to the third order in the order parameters [using the Poisson identity $(1 / 2 E) \tanh (\beta E / 2) \equiv T \Sigma_{n}\left(\omega_{n}^{2}+E^{2}\right)^{-1}$ with $\omega_{n}$ being the Fermion Matsubara frequency], we obtain the GL equations in the uniform case,

$$
\begin{aligned}
0= & -N(0) V_{s}\left\{2 \alpha_{S} S+\gamma\left[2|S|^{2} S+|D|^{2} S+\left|D^{\prime}\right|^{2} S\right.\right. \\
& \left.\left.+\left(S^{*} D+\text { c.c }\right) D+\left(S^{*} D^{\prime}+\text { c.c. }\right) D^{\prime}\right]\right\}, \\
0= & N(0) V_{x^{2}-y^{2}}\left\{\alpha_{D} D+\gamma\left[\frac{3}{4}|D|^{2} D+\frac{1}{4}\left|D^{\prime}\right|^{2} D+|S|^{2} D\right.\right. \\
& \left.\left.+\left(S^{*} D+\text { c.c. }\right) S+\frac{1}{4}\left(D^{*} D^{\prime}+\text { c.c. }\right) D^{\prime}\right]\right\}, \\
0=- & N(0) V_{x y}\left\{\alpha_{D^{\prime}} D^{\prime}+\gamma\left[\frac{3}{4}\left|D^{\prime}\right|^{2} D^{\prime}+\frac{1}{4}|D|^{2} D^{\prime}+|S|^{2} D^{\prime}\right.\right. \\
+ & \left.\left.\left(S^{*} D^{\prime}+\text { c.c. }\right) S+\frac{1}{4}\left(D^{*} D^{\prime}+\text { c.c. }\right) D\right]\right\} .
\end{aligned}
$$

Here $N(0)$ is the normal-state density of states (DOS) at the Fermi surface, $\alpha_{i}=\ln T / T_{i}\left(i=S, D, D^{\prime}\right)$ with $T_{i}$ the bare critical temperatures at the $i$ channel: $T_{S}$ $\approx 1.14 \omega_{c} \exp \left[-1 / N(0) V_{s}\right]$, and $T_{D, D^{\prime}} \approx 1.14 \omega_{c} \exp [-2 /$ $\left.N(0) V_{x^{2}-y^{2}, x y}\right]$ (with $\omega_{c}$ the energy cutoff for the pairing interaction). Finally $\gamma=\int_{-\omega_{c}}^{\omega_{c}} d \epsilon T \Sigma_{n}\left(\omega_{n}^{2}+\epsilon^{2}\right)^{-2}$. On the other hand, the rigidity of the order parameters can be probed by the long-wavelength $\left(\gg \lambda_{F}\right.$ ) spatial variation of the order parameters. For this purpose, it suffices to consider the linearized gap equation but with a small center-of-mass momentum $q\left(\ll k_{F}\right)$ for each Cooper pair,

$$
\Delta_{k, q}=\sum_{k^{\prime}} \frac{V_{k, k^{\prime}} \Delta_{k^{\prime}, q}}{\epsilon_{k_{+}^{\prime}}+\epsilon_{k_{-}^{\prime}}}\left[1-f\left(\epsilon_{k_{+}^{\prime}}\right)-f\left(\epsilon_{k_{-}^{\prime}}\right)\right],
$$

where $k_{ \pm}^{\prime}=k^{\prime} \pm q / 2$, and $f(\epsilon)$ is the Fermi distribution function. We now assume $\Delta_{k, q}=S_{q}+D_{q} \cos 2 \theta_{k}+D_{q}^{\prime} \sin 2 \theta_{k}$, and expand the above equation (again using the Poisson identity) to the second order in $q$ to find that the Fourier components $S_{q}, D_{q}$, and $D_{q}^{\prime}$ satisfy

$$
\begin{gathered}
0=-N(0) V_{s}\left\{2 \alpha_{S} S_{q}+K\left[2 q^{2} S_{q}+\left(q_{x}^{2}-q_{y}^{2}\right) D_{q}\right.\right. \\
\left.\left.+\left(q_{x} q_{y}+q_{y} q_{x}\right) D_{q}^{\prime}\right]\right\}, \\
0=-N(0) V_{x^{2}-y^{2}}\left\{\alpha_{D} D_{q}+K\left[q^{2} D_{q}+\left(q_{x}^{2}-q_{y}^{2}\right) S_{q}\right]\right\}, \\
0=-N(0) V_{x y}\left\{\alpha_{D^{\prime}} D_{q}^{\prime}+K\left[q^{2} D_{q}^{\prime}+\left(q_{x} q_{y}+q_{y} q_{x}\right) S_{q}\right]\right\},
\end{gathered}
$$

where $K=\mathrm{v}_{F}^{2} \gamma / 2$ reflects the rigidity of the order parameters. Resorting to real space, and using the gauge-invariant gradient operator $\Pi=-i \nabla-2 e A$ (with $A$ the vector potential) in substitution of $-i \nabla$, and finally combining the nonlinear homogeneous terms in Eq. (4), we obtain the complete GL equations (up to the third order in the order parameters) describing pairing in all of the three most relevant spin-singlet channels,

$$
\begin{aligned}
& 2 \alpha_{S} S+\gamma\left[2|S|^{2} S+|D|^{2} S+\left|D^{\prime}\right|^{2} S+\left(S^{*} D+\text { c.c. }\right) D\right. \\
&\left.+\left(S^{*} D^{\prime}+\text { c.c. }\right) D^{\prime}\right]+K\left[2 \Pi^{2} S+\left(\Pi_{x}^{2}-\Pi_{y}^{2}\right) D\right. \\
&\left.+\left(\Pi_{x} \Pi_{y}+\Pi_{y} \Pi_{x}\right) D^{\prime}\right]=0, \\
& \alpha_{D} D+\gamma\left[\frac{3}{4}|D|^{2} D+\frac{1}{4}\left|D^{\prime}\right|^{2} D+|S|^{2} D+\left(S^{*} D+\text { c.c. }\right) S\right. \\
&\left.+\frac{1}{4}\left(D^{*} D^{\prime}+\text { c.c. }\right) D^{\prime}\right]+K\left[\Pi^{2} D+\left(\Pi_{x}^{2}-\Pi_{y}^{2}\right) S\right]=0, \quad(5) \\
& \alpha_{D}^{\prime} D^{\prime}+\gamma\left[\frac{3}{4}\left|D^{\prime}\right|{ }^{2} D^{\prime}+\frac{1}{4}|D|^{2} D^{\prime}+|S|^{2} D^{\prime}+\left(S^{*} D^{\prime}+\text { c.c. }\right) S\right. \\
&\left.\quad+\left(D^{*} D^{\prime}+\text { c.c. }\right) D\right]+K\left[\Pi^{2} D^{\prime}+\left(\Pi_{x} \Pi_{y}+\Pi_{y} \Pi_{x}\right) S\right]=0 .
\end{aligned}
$$

For completeness and general purposes, we need a GL freeenergy functional. Following from Eqs. (5), it is given by ${ }^{9}$

$$
\begin{aligned}
F= & \frac{N(0)}{2} \int_{\Omega} 2 \alpha_{S}|S|^{2}+\alpha_{D}|D|^{2}+\alpha_{D^{\prime}}\left|D^{\prime}\right|^{2}+\gamma\left[|S|^{4}+\frac{3}{8}|D|^{4}\right. \\
& +\frac{3}{8}\left|D^{\prime}\right|^{4}+|S|^{2}|D|^{2}+|S|^{2}\left|D^{\prime}\right|^{2}+\frac{1}{4}|D|^{2}\left|D^{\prime}\right|^{2} \\
& \left.+\frac{1}{2}\left(S^{*} D+\text { c.c. }\right)^{2}+\frac{1}{2}\left(S^{*} D^{\prime}+\text { c.c. }\right)^{2}+\frac{1}{8}\left(D^{*} D^{\prime}+\text { c.c. }\right)^{2}\right] \\
& +K\left(2|\Pi S|^{2}+|\Pi D|^{2}+\left|\Pi D^{\prime}\right|^{2}\right) \\
& +K\left\{\left(\Pi_{x} \hat{x}+i \Pi_{y} \hat{y}\right) D \cdot\left[\left(\Pi_{x} \hat{x}-i \Pi_{y} \hat{y}\right) S\right]^{*}+\text { c.c. }\right\} \\
& +K\left\{( \frac { \Pi _ { x } + \Pi _ { y } } { \sqrt { 2 } } \hat { x } + i \frac { \Pi _ { x } - \Pi _ { y } } { \sqrt { 2 } } \hat { y } ) D ^ { \prime } \left[\left(\frac{\Pi_{x}+\Pi_{y}}{\sqrt{2}} \hat{x}\right.\right.\right. \\
& \left.\left.\left.-i \frac{\Pi_{x}-\Pi_{y}}{\sqrt{2}} \hat{y}\right) S\right]^{*}+\text { c.c. }\right\}+\int_{\Omega}(\nabla \times A)^{2} / 8 \pi,
\end{aligned}
$$

where uniformity along the $c$ axis is assumed, and $\int_{\Omega}$ denotes integration over the $a b$ plane. The prefactor of $N(0) / 2$ could be obtained from a microscopic derivation of the su- 
percurrent, but it can also be obtained by the inspection that the free energy reduces to the superconducting ground-state energy at zero temperature. The last term in $F$ is the magnetic energy. The equilibrium supercurrent can now be easily obtained from the functional derivative of the free energy with respect to the vector potential $\delta F / \delta A=0$. It can be seen clearly that the GL equations (or the free-energy functional) would be identical to that derived previously for $d_{x^{2}-y^{2}}$ $+i s$ superconductors if the $d_{x y}$ channel were shut down, and would be equivalent to that investigated for $d_{x^{2}-y^{2}}+i d_{x y}$ superconductors if the $s$-wave channel were disregarded. Moreover, the gradient terms for $D$ and $D^{\prime}$ are symmetric with respect to each other under a rotation of $\pi / 4$.

\section{III. $d_{x^{2}-y^{2}}+i$ is SUPERCONDUCTORS}

This case has been studied previously, ${ }^{10-13}$ so that we shall first recapitulate some essential points that interest us, and add some novel discussions.

We assume that $T_{D}>T_{S}$ as is the case in high- $T_{c}$ superconductors. Define $\alpha_{S D}=\alpha_{S} /\left|\alpha_{D}\right|$ with $\alpha_{D}<0$. Optimizing the free energy Eq. (6) one finds that the uniform bulk phase below the highest bare critical temperature $T_{D}$ is a pure $D$ phase at $\alpha_{S D}>-2 / 3$, or above a critical temperature $T_{S D}$ $=T_{S}^{3} / T_{D}^{2}$, at which the system undergoes a second-order phase transition to a $\top$-broken phase $(S, D) \propto( \pm i \eta, 1)$, with $\eta=|S| /|D|=\sqrt{-\left[3 \alpha_{S D}+2\right) /\left(4+2 \alpha_{S D}\right]}$.

Although there is no uniform $S$ at $T>T_{S D}, S$ can be induced by inhomogeneities, such as surfaces (or twin boundaries), disorders, and vortices, due to the mixed-gradient terms in Eq. (6). Of special interest is the $S$ component generated by vortices. At $T>T_{S D}$, the $S$ component is subdominant and vanishes where $D$ is uniform (e.g., far from the vortex core). Thus it can be obtained perturbatively. Assume $D \sim e^{i \theta}$ in cylindrical coordinates and in the gauge $A$ $=A(r) \hat{\theta}$. Since $\Pi_{x}^{2}-\Pi_{y}^{2}$ transforms as $e^{ \pm 2 i \theta}$ and scales as $1 / r^{2}$ at $r \rightarrow \infty$, we immediately see that to the first order $S$ $\sim a(r) e^{3 i \theta}+b(r) e^{-i \theta}$ [the concrete forms of $a(r)$ and $b(r)$ are unimportant, except that both $a(r)$ and $b(r)$ vanish as $r$ and $1 / r^{2}$ as $r \rightarrow 0$ and $\infty$, respectively] so that $|S|^{2} \sim a(r)^{2}$ $+b(r)^{2}+2 a(r) b(r) \cos 4 \theta$. (For complex $a$ and $b$, there are $\theta$-independent phase shifts in the argument of the cosine function, but they do not alter our general conclusion. This is also the case in similar discussions hereafter). Therefore $|S|^{2}$ is fourfold symmetric near the vortex core and vanishes far from the core. This result has been obtained previously by many authors, ${ }^{10-12,14}$ in different contexts, and has been verified by our numerical simulations. ${ }^{13,15}$ In particular, Franz et al. were able to obtain explicit analytical as well as numerical solutions for the single vortex state, ${ }^{12}$ which is useful for a quantitative comparison between the theory and the experiments. The relative phase of these two components varies continuously around the vortex core. However, such a kind of vortex-induced $S$ is insufficient, at least at low fields, to change the bulk quasiparticle DOS $N(\omega)$ $\sim 1 / \Omega \int_{\Omega} \Sigma_{k} \delta\left(\omega-\sqrt{\epsilon_{k}^{2}+\left|\Delta_{k}\right|^{2}}\right)$ in that the induced $S$ component is localized near the vortex core. Moreover, the behavior of $S$ does not show any sign of increasing sensitivity to the applied field at decreasing temperatures. Therefore, we may rule out the role of the $s$ channel in the abnormal ther- mal conductivity of Krishana et $a .^{4}$

On the other hand, at $T<T_{S D}, \top$ is already broken in the uniform phase. Lowest-order perturbative treatment of $S$ is no longer valid at $T \ll T_{S D} . S$ and $D$ are now of competing order far from the core with a locked relative phase $\pm \pi / 2$ because of the fact that they are uniform there. In other words, symmetry consistency requires that $S$ develops a winding component $\sim e^{i \theta}$ in addition to the $e^{-i \theta}$ and $e^{3 i \theta}$ components, i.e., $S \sim a e^{i \theta}+b e^{3 i \theta}+c e^{-i \theta}+$ higher-order terms near the vortex core. Thus generally $|S|^{2}=a^{2}+b^{2}$ $+c^{2}+2(a b+a c) \cos 2 \theta+2 b c \cos 4 \theta$, developing a striking twofold, rather than a fourfold, symmetry near the vortex core. The situation is similar for the $D$ component. The relative phase varies again near and around the vortex, but is locked to $\pm \pi / 2$ far away from the core. As the twofold symmetry is generated solely from $a e^{i \theta}$, the quantity $a(r$ $\rightarrow \infty)= \pm i \eta$ can also be thought of as the order parameter of the twofold-symmetric vortex structure. Recent numerical calculations support the scenario that a structure phase transition for the vortex profile convolute with the uniform $\top$-breaking phase transition. ${ }^{13}$ Such an exotic vortex can possibly be observed either by magneto-optical spectroscopy, or more possibly by its response to a "rotating" applied current $\mathbf{J}=J(\cos \omega t \hat{x}+\sin \omega t \hat{y})$. At low fields and in a high-quality crystal, there should be a resonance in the vortex dissipation at a frequency comparable to the scale of the energy barrier for the vortex to rotate by an angle of $\pi / 2$ or $\pi$.

The mixing of the two order parameters $S$ and $D$ gives a nontrivial upper critical field, $B_{c 2}$. In our case, the linearized GL equations are

$$
\begin{gathered}
2 \alpha_{S} S+K\left[2 \Pi^{2} S+\left(\Pi_{x}^{2}-\Pi_{y}^{2}\right) D\right]=E S, \\
\alpha_{D} D+K\left[\Pi^{2} D+\left(\Pi_{x}^{2}-\Pi_{y}^{2}\right) S\right]=E D
\end{gathered}
$$

where we have added an eigenenergy term $E$ on the righthand side of the equations. $E=0$ corresponds to the solution of the GL equations. The condition that the ground-state energy $E_{g}=0$ determines the upper critical field. This problem has been treated in Refs. 11 and 12 where an implicit solution for $B_{c 2}$ was obtained. Here we give an explicit solution along the line of Sigrist and Veda. ${ }^{16}$ By the inspection that $\left[\Pi_{x}, \Pi_{y}\right]=2 i e B$, we can define the bosonic operators $a$ and $a^{\dagger}$ such that $\left[a, a^{\dagger}\right]=1$, with

$$
a=\left(\Pi_{x}+i \Pi_{y}\right) / \sqrt{4 e B}, \quad a^{\dagger}=\left(\Pi_{x}-i \Pi_{y}\right) / \sqrt{4 e B} .
$$

Here $B$ is the magnetic induction, the fluctuation of which at the upper critical field can be neglected. In terms of the bosonic operators, the above linearized GL equations become

$$
\begin{aligned}
& {\left[4 K e B(2 \hat{n}+1)+2 \alpha_{S}\right] S+2 K e B\left(a a+a^{\dagger} a^{\dagger}\right) D=E S,} \\
& 2 K e B\left(a a+a^{\dagger} a^{\dagger}\right) S+\left[2 K e B(2 \hat{n}+1)+\alpha_{D}\right] D=E D .
\end{aligned}
$$

Here $\hat{n}=a^{\dagger} a$ is the Landau quantum number. $S$ and $D$ can be expanded in terms of the Landau levels $|n\rangle$ as $(S, D)$ $=\Sigma_{n}\left(a_{n}, b_{n}\right)|n\rangle$. If there were no coupling between $S$ and $D$, we would obtain the usual result that the lowest Landau level $n=0$ determines the upper critical field. In our case, how- 
ever, the eigenvalue problem involves coupling between all the next-nearest Landau levels. Expanding the order parameters up to $n=2$, we can find the ground-state energy $E_{g}$ variationally. The upper critical field is obtained by setting $E_{g}=0$, and is $B_{c 2}=\left[5-\alpha_{S D}+\sqrt{\left(\alpha_{S D}^{2}+6 \alpha_{S D}+25\right)}\right] B_{0} / 8$ with $B_{0}=-\alpha_{D} / 2 e K$ being the temperature-dependent bare upper critical field for the pure $d_{x^{2}-y^{2}}$ superconductors. In full dimensions, $B_{0}=-\alpha_{D} \hbar c / 2 e K=\Phi_{0} / 2 \pi \xi^{2}$ with $\Phi_{0}$ $=2 \pi \hbar c / 2 e=h c / 2 e$ and $\xi^{2}=-K / \alpha_{D}$. It should be pointed out that the above variational ground state is closely similar to that obtained in Refs. 11 and 12, where $S$ are given by a difference between two Gaussian functions. This is clear from the fact that $S$ has two nodes in such a case, as the Landau state with $n=2$ does. Indeed, these solutions behave similarly: $B_{c 2} \rightarrow B_{0}$ at $T \rightarrow T_{D}$ (or $\alpha_{S D} \rightarrow \infty$ ), but it is always larger than $B_{0}$, developing an upward curvature in the temperature dependence near and below $T_{D} \cdot{ }^{11,12}$ (It diverges in the unphysical region $\alpha \rightarrow-\infty$.) This is due to the very effect that the subdominant channel contributes excess energy lowering by adjusting the shape of the vortex.

Finally, the Abrikosov vortex lattice has also been addressed in the literature. ${ }^{10-13}$ The nontrivial fourfold or twofold vortex structures have important impact on the lattice structure. Due to the intrinsic anisotropies arising from $|S|^{2}$ they favor generally an oblique vortex lattice. ${ }^{10-13}$ Of course, with increasing temperatures, the amplitude of $S$ decreases, and one generally expects a crossover to the hexagonal vortex lattice near $T_{D}$. Another way of seeing this is as follows. Since the vortex lattice can be constructed in terms of the (highly degenerated) ground-state wave functions for the linearized GL equations, it cannot be strictly hexagonal if the amplitude of the $n=2$ Landau level is finite. ${ }^{16}$ Franz et al. were also able to conclude that the orientational angle of the vortex lattice with respect to the crystal axis $\alpha$ is no longer arbitrary (as in the case of a single order parameter), but can only take the four possible values $\alpha= \pm \pi / 2, \pm \pi,{ }^{12}$ as was found in numerical simulations. ${ }^{15}$ In the limit of $\alpha_{S D} \rightarrow \infty$ (or $\left.T \rightarrow T_{D}\right)$, however, the amplitude for $|n=2\rangle$ vanishes and we recover the usual result for a pure $D$ order parameter.

Before closing this section, we would like to point out that all conclusions drawn for $d_{x^{2}-y^{2}}+i$ s superconductors are also true for the $d_{x y}+i s$ superconductors due to the apparent symmetry.

\section{IV. $d_{x^{2}-y^{2}}+i d_{x y}$ SUPERCONDUCTORS}

We consider the case $T_{D}>T_{D^{\prime}}$. Similar to the above case, there is also a second-order $T$-breaking phase transition. Here the transition temperature $T_{D D^{\prime}}$ is given by $\alpha_{D^{\prime} D} \equiv \alpha_{D^{\prime}} /\left|\alpha_{D}\right|<-1 / 3 \quad$ (with $\quad \alpha_{D}<0$ ), or $T_{D^{\prime} D}$ $=\sqrt{T_{D^{\prime}}^{3} / T_{D}}$. Below $T_{D D^{\prime}}$ and in the uniform phase, $D^{\prime}$ $= \pm i \eta D$ with $\eta=\sqrt{-\left(1+3 \alpha_{D^{\prime} D}\right) /\left(3+\alpha_{D^{\prime} D}\right)}$. However, above $T_{D D^{\prime}}$ no $D^{\prime}$ component can be induced by a vortex in the absence of a mixed-gradient term coupling $D^{\prime}$ with $D$. Below $T_{D D^{\prime}}$, symmetry requires that both components wind in the same manner, e.g., $D, D^{\prime} \sim e^{i \theta}$. Also because of this, the upper critical field is independent of $\alpha_{D^{\prime} D}$ so long as $\alpha_{D^{\prime} D}>-1$ (or simply $T>0$ ). In this case, $D^{\prime} / D=0$ at the upper critical field, even though $D^{\prime} \neq 0$ in the absence of the field.
In fact, because $D$ and $D^{\prime}$ are not coupled by gradients, the system has two metastable states with $D^{\prime}=0$ and $D^{\prime}$ $\neq 0$, respectively. The stable state is that with lower free energy. This is related to the fact that the $T$-breaking phase is frustrated at the vortex core if $\alpha_{D^{\prime} D}>-1$ : While winding simultaneously for $D$ and $D^{\prime}$ increases the kinetic energy of the superfluid, the nonzero $D^{\prime}$-component may lower the homogeneous energy at low fields. We believe the competing energies may drive a transition from the $d_{x^{2}-y^{2}}+i d_{x y}$-wave vortex state to a pure $d_{x^{2}-y^{2} \text {-wave vortex }}$ state at a field lower than the upper critical field. By the observation that the system is in the $d_{x^{2}-y^{2}}+i d_{x y}$-wave phase at zero field but is not at the upper critical field, and that the system would be in the $d_{x^{2}-y^{2}}+i d_{x y}$-wave vortex state at all fields when $\alpha_{D^{\prime} D}=-1$, there must be at least one field-induced phase transition below $T_{D D^{\prime}}$. The transition field is zero and $B_{c 2}$ for $T=T_{D D^{\prime}}$ and $T=0$, respectively, and should generally increase with decreasing temperature. Above the transition line is the region of pure $d_{x^{2}-y^{2}}$ mixed state. Such a transition would predict that the quasiparticle excitation gap (which should be proportional to $\left|D^{\prime}\right|$ ) decreases with increasing field below the transition line, and eventually vanishes above the transition line, where residual density of states can arise from the Doppler-shift due to the supercurrent around the vortex. ${ }^{5,17}$ (The field-induced lowlying quasiparticle states are possible only for a nodal pairing function.) This translates that at $T<T_{D D^{\prime}}$ the thermal conductivity should increase rather than decrease with increasing field, being consistent with the general trend of the data at sub-Kelvin temperatures in Ref. 5. But the concrete field dependence of the thermal conductivity may be different. In fact, the mechanism proposed in Ref. 5 rules out the $D^{\prime}$ component.

\section{STRONG COUPLING EFFECTS: PHENOMENOLOGICAL THEORY}

As the electrons in unconventional superconductors are strongly correlated because of their $d$-shell character, we now incorporate the strong-coupling effects in a pure phenomenological manner by including the Zeeman energy. This energy follows from the interaction between the magnetic field and the angular momentum of the Cooper pair via strong-coupling effects. The underlying mechanism is simple: Cooper pairs with a definite projection of internal orbital momentum will respond to the direction of the vortex supercurrent, which is determined by the magnetic field. ${ }^{18}$ The desired energy density is ${ }^{16}$

$$
-4 \pi e h \int d^{2} \mathbf{R} g(R) \Delta_{\mathbf{R}}^{*} \mathbf{R} \times \frac{\nabla_{\mathbf{R}}}{i} \Delta_{\mathbf{R}} \propto i h\left(D^{*} D^{\prime}-\text { c.c. }\right),
$$

where $h=\nabla A$ is the local magnetic induction, $g(R)$ is representative of the strong-coupling effect, and $\Delta_{\mathbf{R}}$ is the Cooper-pair wave function in the center-of-mass frame, i.e., the inverse Fourier transform of $\Delta_{k}=S+D \cos 2 \theta_{k}$ $+D^{\prime} \sin 2 \theta_{k}$ subject to the weak-coupling condition $|k|$ $=k_{F}$,

$$
\begin{aligned}
\Delta_{\mathbf{R}} \sim & \int \Delta_{k} e^{i \mathbf{k} \cdot \mathbf{R}} d \theta_{k}=S_{J_{0}(k R)}+\left(D \cos 2 \theta_{R}\right. \\
& \left.+D^{\prime} \sin 2 \theta_{R}\right) J_{2}(k R),
\end{aligned}
$$


with $J_{n}(k R)=\int \cos n \phi e^{i k R \cos \phi} d \phi$, and $\theta_{R}$ being the angle between $\mathbf{R}$ and the $a$ axis in the copper-oxide plane. Here we have adopted the local limit by neglecting the spatial variation of the order parameters in evaluating the strongcoupling term, since $\lambda_{F} \ll \xi$, with $\xi$ being the coherence length of the superconductor. Note that the final result of the strong-coupling term does not include the $S$ component. This is not accidental but results from symmetry. For convenience we rewrite the nondiamagnetic contribution (7) in the form as $\delta k K(2 i e h)\left(D^{*} D^{\prime}-\right.$ c.c. $)$ with the only phenomenological dimensionless parameter $\delta k$. Notice that the Zeeman term can also be rewritten as gradient terms $\delta k K\left[\left(\Pi_{x} D\right) * \Pi_{y} D^{\prime}-\left(\Pi_{y} D\right) * \Pi_{x} D^{\prime}+\right.$ c.c. $]$ due to the fact that $\left[\Pi_{x}, \Pi_{y}\right]=2 i e h$.

To simplify the matter, here we only consider the $d_{x^{2}-y^{2}}+i d_{x y}$ superconductor in the presence of a strongcoupling effect. In this case, the new free energy reads,

$$
\begin{aligned}
F= & \frac{N(0)}{2} \int_{\Omega} \alpha_{D}|D|^{2}+\alpha_{D^{\prime}}\left|D^{\prime}\right|^{2}+\gamma\left[\frac{3}{8}|D|^{4}+\frac{3}{8}\left|D^{\prime}\right|^{4}\right. \\
& \left.+\frac{1}{4}|D|^{2}\left|D^{\prime}\right|^{2}+\frac{1}{8}\left(D^{*} D^{\prime}+\text { c.c. }\right)^{2}\right]+K\left[|\Pi D|^{2}+\left|\Pi D^{\prime}\right|^{2}\right. \\
& \left.+2 i e h \delta k\left(D^{*} D^{\prime}-\text { c.c. }\right)\right]+\int_{\Omega}(\nabla A)^{2} / 8 \pi .
\end{aligned}
$$

In the case of $\alpha_{D} \equiv \alpha_{D^{\prime}}<0$ and $\delta k \neq 0$, it is known that there could be a first-order phase transition from the Meissner state to a state with a sudden penetration of a finite density of vortices at a lower critical field $H_{c 1} \cdot{ }^{19}$ (The transition is of first order since $B_{c 1} \neq 0$ at $H=H_{c 1}$.) However, in the extreme London limit that we shall adopt (suitable for high$T_{c}$ superconductors), we expect that both $H_{c 1}$ and $B_{c 1}$ would be too small to alter our subsequent discussion, and will be neglected.

Let us now discuss the outcome of Eq. (8). To be concrete but without loss of generality, we assume $T_{c}=T_{D}=100 \mathrm{~K}$ and $T_{D^{\prime}}=10 \mathrm{~K}$. As discussed in the previous section, at zero magnetic field, there is a second order $T$-breaking phase transition at a critical temperature $T_{D D^{\prime}}=\sqrt{T_{D^{\prime}}^{3} / T_{D}} \approx 3.16 \mathrm{~K}$. Below $\quad T_{D D^{\prime}}, \quad D^{\prime}= \pm i \eta D . \quad$ Explicitly, $\quad|D|^{2}=(3$ $\left.+\alpha_{D^{\prime} D}\right) D_{0}^{2} / 2$ and $\left|D^{\prime}\right|^{2}=-\left(1+3 \alpha_{D^{\prime} D}\right) D_{0}^{2} / 2$. Here $D_{0}^{2}$ $=-\alpha_{D} / \gamma$. Henceforth we shall normalize the order parameters in units of $D_{0}$, and the induction field by $B_{0}$ defined previously. They are understood as dimensionless quantities unless specified otherwise. Practically for high- $T_{c}$ superconductors, both $D_{0}$ and $B_{0}$ saturate at low temperatures (e.g., $T \leqslant 20 \mathrm{~K}): D_{0} \sim 2 T_{c} \sim 200 \mathrm{~K}$ and $B_{0} \sim B_{c 2}(0) \sim 40-100 \mathrm{~T}^{20}$

Following from Eq. (8), in the presence of the nondiamagnetic coupling between the order parameters and the magnetic field ( $\delta k \neq 0)$, a pure $d_{x^{2}-y^{2}}$ state is stable only in zero field above $T_{D D^{\prime}}$, while a $T$-broken $d_{x^{2}-y^{2}} \pm i d_{x y}$ state is established at all temperatures at finite fields, albeit with varying amplitude of $D^{\prime}$. We now concentrate on the lowfield regime and in the London limit, so that the kinetic energy of the superfluid and the inhomogeneity due to vortices can be safely neglected. The same methodology has been applied in Ref. 6. At $T>T_{D D^{\prime}}$ and low fields, the order parameters can be treated perturbatively, and are, up to the

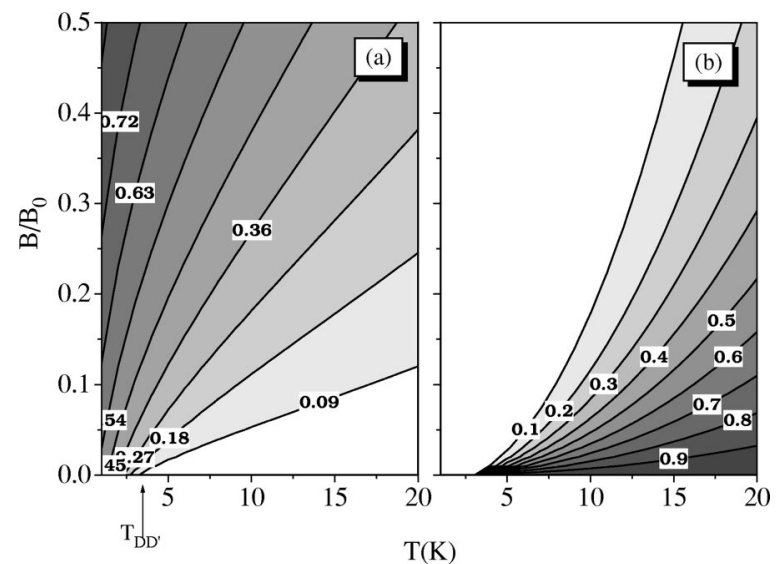

FIG. 1. Gray-scale contour plots of (a) $\left|D^{\prime}\right|$ and (b) $\kappa_{e}(T, B) / \kappa_{e}(T, 0) \approx(1 / T) \exp \left(-\Delta_{0} / T\right)$ as functions of $B$ and $T$. Here $\delta k=1 / 2$ and $\Delta_{0}=2\left|D^{\prime}\right| T_{c}$ with $T_{c}=100 \mathrm{~K} . T_{*} \sim 3.16 \mathrm{~K}$ is the zero-field $T$-breaking transition temperature. See the text for details.

second order in $\epsilon \equiv \delta k B$ ( $>0$ for ease of presentation) with $B$ as the spatial average of the local induction $h$,

$$
\begin{gathered}
|D|=2 / \sqrt{3}-\epsilon\left|D^{\prime}\right| / 4, \\
\left|D^{\prime}\right|=(\epsilon / \sqrt{3}) /\left(\alpha_{D^{\prime} D}+1 / 3\right) .
\end{gathered}
$$

This amounts to a minimum gap on the Fermi surface

$$
\Delta_{0} \approx 2\left|D^{\prime}\right| T_{c} \sim 2 \epsilon T_{D} T_{D^{\prime} D} /\left(T-T_{D^{\prime} D}\right) \propto \delta k B /\left(T-T_{D^{\prime} D}\right)
$$

at $\epsilon \ll 1$ and $T>T_{D^{\prime} D}$ (not very close to $T_{D^{\prime} D}$ ). Neglecting for the moment the suppression due to quasiparticle scattering at the vortices, we observe that the thermal conductivity $\kappa_{e}$ at $T>T_{D^{\prime} D}$ in the presence of a minimum gap $\Delta_{0}$ is given by,

$$
\begin{aligned}
& \kappa_{e}(T, B) / \kappa_{e}(T, 0) \sim(1 / T) \exp \left(-\Delta_{0} / T\right) \\
& \quad \sim(1 / T) \exp \left[-\mathrm{const} \times \delta k B /\left(T-T_{D^{\prime} D}\right) T\right] .
\end{aligned}
$$

It should be appreciated that this exponential decay in $B$ is increasingly sharpened by $T\left(T-T_{D^{\prime} D}\right)$ instead of only $T$. In Eq. (12) a power law $\kappa_{e}(T, 0) \propto T^{2}$ is used, which follows from the fact that above $T_{D^{\prime} D}$ the zero-field state is a pure $d_{x^{2}-y^{2}}$ state. Further we have implicitly neglected the change in $|D|$, which is merely of the second order in $\epsilon$ from Eq. (9). The exponential decay develops a crossover at $\Delta_{0} \sim T$, or,

$$
B_{k} \propto T\left(T-T_{D^{\prime} D}\right),
$$

which indeed resembles a scaling law $B_{k} \propto T^{2}$ addressed by Krishana et al. ${ }^{4}$ As an example, we plot $\left|D^{\prime}\right|$ and $\kappa_{e}(T, B) / \kappa_{e}(T, 0)$ in this context in Figs. 1(a) and 1(b), respectively. Here $\left|D^{\prime}\right|$ is calculated exactly from the optimization of the free-energy subject to the neglecting of kinetic energy and field energy, which should be reliable at low fields. Defining a criterion for the kink transition (more precisely, crossover), the paraboliclike contour lines unambiguously reproduce the scaling law for the kink field. ${ }^{4}$ The sharpening feature of the crossover is also clear. Including 
the additional suppression of $\kappa_{e}$ from quasiparticle scattering by vortices would render even better qualitative agreement with the experimental results, but we shall not go into further details. Interestingly, Eq. (13) predicts a downward curvature of the $B_{k}(T)$ curve in a log-log plot on the low-temperature side, which seems to be the case in Fig. 4(b) of Ref. 4. We have also performed exact calculation of the full set of GL equations ensuing from Eq. (8) to find that the results in the low-field regime remains essentially unchanged, while at intermediate fields, $\left|D^{\prime}\right|$ saturates a while and then diminishes gradually together with $|D|$ near the upper critical field $B_{c 2}$. The latter is given by, in dimensionless form,

$$
\begin{aligned}
B_{c 2}= & {\left[1-\alpha_{D^{\prime} D}+\sqrt{\left(1+\alpha_{D^{\prime} D}\right)^{2}-4 \alpha_{D^{\prime} D} \delta k^{2}}\right] / 2 } \\
& \times\left(1-\delta k^{2}\right) .
\end{aligned}
$$

At $B_{c 2}$ the ratio $\left|D^{\prime}\right| /|D|$ is given by

$$
\left|D^{\prime}\right| /|D|=\delta k B_{c 2} /\left(\alpha_{D^{\prime} D}+B_{c 2}\right) .
$$

This means that $T$ breaking is retained up to the upper critical field. However, it is clear, in conjunction with Fig. 1(a), that the strong-coupling effect vanishes near the critical temperature $T_{c}$ (where $\alpha_{D^{\prime} D} \rightarrow \infty$ ), a necessary ingredient for the theory to be compatible with the general properties of high$T_{c}$ superconductors at temperatures near $T_{c}$. Also interestingly, in the context of this theory, (i) the power law $\kappa_{e}$ $\propto T^{2}$ is invalid at finite fields above $T_{D^{\prime} D}$, which seems to be consistent with the experimental data in Ref. 4, and would be violated below $T_{D^{\prime} D}$ even in the absence of magnetic field; (ii) $\kappa_{e}$ increases with increasing magnetic field below $T_{D^{\prime} D}$. On the other hand, the theory does not involve a phase transition for the development of a plateau in $\kappa_{e}$, because there is no further symmetry breaking at finite fields in our theory.

\section{SUMMARY}

We have developed a GL theory with respect to the three relevant singlet pairing channels in unconventional superconductors. The case of $d+i s$ superconductors has been discussed. The twofold symmetric structure of vortices below the $T$-breaking temperature is predicted. The case of $d_{x^{2}-y^{2}}+i d_{x y}$ superconductors has been addressed in detail. In the absence of strong-coupling effects, we find that the subdominant $d_{x y}$-wave order parameter cannot be induced by vortices above the $T$-breaking temperature, in contrast to the case in a $d+i s$ superconductor. Below the $T$-breaking temperature, we predict a field-induced first-order phase transition from the $d_{x^{2}-y^{2}}+i d_{x y}$ vortex state to the $d_{x^{2}-y^{2}}$ vortex state at higher fields. By including the strong-coupling effect phenomenologically, the $d_{x^{2}-y^{2}}+i d_{x y}$ state can be induced by the magnetic field even above the zero-field $T$-breaking temperature. Near and above this transition temperature, the induced $d_{x y}$-wave order parameter may be compared with some results for the abnormal thermal conductivity reported in Ref. 4. Although our theory could give the general trend that the thermal conductivity would increase with increasing field below the $T$-breaking temperature, it cannot result in a pure $d_{x^{2}-y^{2}}$ state near the zero temperature, which was claimed to be essential to explain the sub-Kelvin thermal conductivity in Refs. 5 and 17. If that is the case, we argue that there are two possibilities for the material used in Ref. 5: either some significant change occurs for the $d_{x y}$ channel near zero temperature such that the $d_{x y}$-wave order parameter is suppressed drastically or no $d_{x y}$ channel is present at all.

It should be pointed out that the $s$ and $d_{x y}$ channels are only two, but not all of the possible subdominant singlet pairing channels in a $d_{x^{2}-y^{2}}$-dominant superconductor. We have neglected, e.g., the $k_{x} k_{y}\left(k_{x}^{2}-k_{y}^{2}\right)$ channel (in the $A_{2 g}$ representation), which would appear in the case of a more general pairing interaction, or in a higher-order expansion of the gap equation. ${ }^{21}$ This order parameter could also be induced by spatial inhomogenieties of the $d_{x^{2}-y^{2}}$ order parameter, and would indirectly induce a $d_{x y}$ order parameter. However, odd-parity order parameters cannot be induced in singlet-pairing superconductors.

In this paper, we have only performed accessible analytical as well as qualitative discussions on the theory. More exact and numerical results are awaited. The theory is also highly useful for studying the vortex dynamics of unconventional superconductors. ${ }^{15}$

\section{ACKNOWLEDGMENTS}

This work was supported by the HKU block grant, the RGC grant of Hong Kong under No. HKU7116/98P and No. HKU 7144/99P, the National Natural Science Foundation of China, and supported, in part, by the Sanzhu Co. Ltd. in Shandong through a Ke-Li Fellowship. Z.D.W. acknowledges the hospitality of the National Center for Theoretical Sciences at Taiwan, where the final version was completed.
*Electronic address: zwang@hkucc.hku.hk

${ }^{1}$ C. C. Tsuei et al., Phys. Rev. Lett. 73, 593 (1997); J. R. Kirtley et al., Nature (London) 373, 225 (1995).

${ }^{2}$ M. Covington et al., Phys. Rev. Lett. 79, 277 (1997); M. Fogelström, D. Rainer, and J. A. Sauls, ibid. 79, 281 (1997).

${ }^{3}$ J. R. Kirtley et al., Phys. Rev. B 51, 12057 (1995). For a theoretical interpretation, see, e.g., M. Sigrist, D. B. Bailey, and R. B. Laughlin, Phys. Rev. Lett. 74, 3249 (1995).

${ }^{4}$ K. Krishana et al., Science 277, 83 (1997).

${ }^{5}$ H. Aubin, K. Behnia, S. Ooi, and T. Tamegai, Phys. Rev. Lett. 82, 624 (1999).

${ }^{6}$ R. B. Laughlin, Phys. Rev. Lett. 80, 5188 (1998).

${ }^{7}$ H. Ghosh, Europhys. Lett. 43, 707 (1998); Phys. Rev. B 59, 3357 (1999); cond-mat/9904375 (unpublished).
${ }^{8}$ K. A. Musaelian, J. Betouras, A. V. Chubukov, and R. Joynt, Phys. Rev. B 53, 3598 (1996); M. Liu, D. Y. Xing, and Z. D. Wang, ibid. 55, 3181 (1997).

${ }^{9}$ It is understood that the temperature dependencies in the parameters of the free-energy functional is valid strictly near the superconducting temperature. At low temperatures, $\gamma$ and $K$ can be shown to be roughly temperature independent from more exact analysis. On the other hand, $\alpha_{i}$ is better approximated as $\alpha_{i}$ $\sim\left(T-T_{i}\right) / T_{i}$ at low temperatures in order to reproduce the fact that $\Delta_{k}$ saturates as $T \rightarrow 0$. However, these complications are not crucial for our main conclusions in the text.

${ }^{10}$ Y. Ren, J. H. Xu, and C. S. Ting, Phys. Rev. Lett. 74, 3680 (1995).

${ }^{11}$ A. J. Berlinsky, A. L. Fetter, M. Franz, C. Kallin, and P. I. 
Soininen, Phys. Rev. Lett. 75, 2200 (1995).

${ }^{12}$ M. Franz, C. Kallin, P. I. Soininen, A. J. Berlinsky, and A. L. Fetter, Phys. Rev. B 53, 5795 (1996).

${ }^{13}$ Q. Li, Z. D. Wang, and Q.-H. Wang, Phys. Rev. B 59, 613 (1999); 60, 14577 (1999).

${ }^{14}$ G. E. Volovik, Pis'ma Zh. Éksp. Teor. Fiz. 58, 457 (1993) [JETP Lett. 58, 469 (1993)].

${ }^{15}$ Q. Wang and Z. D. Wang, Phys. Rev. B 54, R15 645 (1996); Z. D. Wang and Q. H. Wang, ibid. 55, 11756 (1997).
${ }^{16}$ M. Sigrist and K. Ueda, Rev. Mod. Phys. 63, 239 (1991).

${ }^{17}$ C. Kübert and P. Hirschfeld, Phys. Rev. Lett. 80, 4963 (1998).

${ }^{18}$ T. A. Tokuyasu, D. W. Hess, and J. A. Sauls, Phys. Rev. B 41, 8891 (1990)

${ }^{19}$ Z. D. Wang and Q. H. Wang, Phys. Rev. B 57, R724 (1998); Q. H. Wang and Z. D. Wang, ibid. 57, 10307 (1998).

${ }^{20}$ C. Panagopoulos and T. Xiang, Phys. Rev. Lett. 81, 2336 (1998).

${ }^{21}$ Wonkee Kim, Jian-Xin Zhu, and C. S. Ting, Phys. Rev. B 57, 13 403 (1998) 